\title{
New experimental markers for early detection of high-risk prostate cancer: role of cell-cell adhesion and cell migration
}

\author{
A. J. M. Mol • A. A. Geldof • G. A. Meijer • \\ H. G. van der Poel · R. J. A. van Moorselaar
}

Received: 2 March 2007 / Accepted: 26 April 2007 / Published online: 23 May 2007

(C) Springer-Verlag 2007

\begin{abstract}
Today's treatment and diagnosis of prostate cancer still exhibit major limitations. The search for new and additional prognostic markers is therefore still an actual field of interest. Potential markers involved in numerous biological processes in the tumor cell have been investigated intensively. For therapeutic interventions it is important to distinguish between harmless and aggressive disease in an early stage. Therefore the subject of this review is limited to markers associated with those functional processes, which discriminate early stage aggressive, metastatic cancer from harmless disease. Important processes in this respect are: altered cell adhesion and cellular migration. E-cadherin, $\mathrm{N}$-cadherin, $\beta$-catenin, integrins, focal adhesion kinase, connexins and matrix metalloproteinases all appear promising biological markers associated with the early stage metastatic process in prostate cancer. Here we discuss their potential to become valid biological markers based on literature data. Thus far, none of these markers proved to be a valid individual marker by itself due to
\end{abstract}

A. J. M. Mol ( $ه)$ · A. A. Geldof · R. J. A. van Moorselaar Department of Urology, VU University Medical Center, PO Box 7057, 1007, MB Amsterdam, The Netherlands e-mail: A.mol@VUMC.nl

\section{A. A. Geldof}

Department of Nuclear Medicine and PET Research,

VU University Medical Center, PO Box 7057,

1007, MB Amsterdam, The Netherlands

\section{G. A. Meijer}

Department of Pathology, VU University Medical Center, PO Box 7057, 1007, MB Amsterdam, The Netherlands

H. G. van der Poel

Department of Urology, Netherlands Cancer Institute, Plesmanlaan 121, 1066, CX Amsterdam, The Netherlands prostate cancer heterogeneity and transient expression. Analyzing a combination of the potential markers discussed in this review is expected to be a better approach toward discriminating high- from low-risk tumors in an early stage of prostate cancer.

Keywords Prostatic neoplasms · Tumor markers .

Biological · Prognosis $\cdot$ Neoplasm metastasis .

Neoplasm invasiveness · Cell adhesion .

Intercellular junctions $\cdot$ Review

\section{Introduction}

Prostate cancer (PC) is the most frequent cancer in men of western countries (Crawford 2003). Screening for prostate specific antigen (PSA) in asymptomatic populations has increased the detection rate of prostate cancer as well as the number of surgical and radiotherapeutic treatments. Recently, the use of PSA as a diagnostic marker has come to be discussed (Stamey et al. 2002). An elevated level of serum PSA not only detects aggressive malignancy but it is also associated with benign prostate hyperplasia (BPH) and with mildly aggressive, slowly progressing, neoplasia. Furthermore, aggressive and metastatic prostate tumors have been identified which showed no elevated serum level of PSA. In addition, approximately $86 \%$ of men diagnosed with PC are not destined to die from the disease (Klotz 2006).

A large proportion of prostate carcinomas remain confined in the prostate, while only a small proportion of carcinomas acquire the ability to metastasize. Pathological grading using the Gleason score, together with PSA measurement is still insufficient to distinguish between indolent tumors and aggressive metastatic tumors. Gleason score is 
one of the most accepted and widely used grading systems for prostate carcinoma. More aggressive disease is associated with higher Gleason scores. The Gleason score is a summation of the two most prevalent Gleason grades (1-5). This reflects tumor heterogeneity often present in prostate cancer. Subpopulations of tumor cells within the tumor, therefore, should be identified in an early phase of disease management enabling adequate prediction of tumor behavior.

Low-grade tumors, as defined by pathological examination of biopsies, are usually left untreated. A watchful waiting approach may be adopted till progressing carcinoma is suspected. Based on serum PSA level, number of tumorcontaining core-biopsies and Gleason score of the tumor, patients may be selected for a wait and see approach. Rising PSA levels, PSA doubling time and local tumor growth are indicators that a tumor is progressing. At present, adequate prognostic or predictive markers for tumor progression are still lacking. Although PSA combined with pathological grading is still considered as the most reliable diagnostic marker today, the number of false positives and, therefore, unnecessary treatments indicate a need for new, or additional, prognostic markers, which are able to distinguish between indolent and aggressive prostate carcinomas at an early stage. Here we select potential markers that may be used to distinguish between indolent and aggressive tumors at an early stage, based on biological function and expression in prostate carcinoma.

\section{Marker selection criteria}

\section{Early stage}

A suitable marker should distinguish normal tissue from carcinoma. Prostate cancer usually occurs in elderly men. Low aggressive carcinomas do not always need treatment while patients often die from other factors than the prostate cancer itself. So, in addition, it would be optimal if the marker were able to differentiate between low and high aggressive carcinomas. As treatment is most successful at early stages of the disease, a prognostic marker should distinguish between low- and high aggressive carcinoma as early as possible.

\section{Biological processes involved in metastasis}

Early stage disease differs from later stages in tumor volume, localization and metastatic potential. The first difference between high and low aggressive carcinomas is its potential to metastasize. Therefore, an early discrimination could be made on the base of the biological processes at work in the epithelial cells, which lead to metastatic potential.
Other biological processes involved in tumor progression are less likely to distinguish high- from low aggressive disease in an early stage. For example; cell proliferation is disturbed in both low grade and early-stage diseases; this process is not likely to involve a protein, which could distinguish high- from low aggressive carcinomas. Furthermore, processes involved in later stage diseases, like developing androgen independency as a consequence of androgen depletion therapy, homing of metastatic cells in lymphatic or bone tissue and neoangiogenesis cannot be detected in early stage disease. Proteins involved mainly in those processes are therefore not likely to be adequate markers in early stage disease and will not be subject of this review. Here potential markers are discussed based on their biological function in the early metastatic process as this may reflect future tumor behavior in early stage disease.

\section{Change in expression}

Although biological function is important, a molecule's role in tumorigenesis is not a license to become a valid marker. A change in expression, or expression pattern, may be sufficient to detect malignancy. However, an optimal marker must be representative for disease progression. This means that the expression of the candidate marker must be proportional to, or inversely related to, disease progression. Most convincing would be a marker, which is overexpressed in tissues developing into aggressive carcinoma. As it would be optimal to distinguish aggressive carcinoma from indolent disease as early as possible, a change in expression compared to normal tissue must be observable in an early stage of disease.

\section{Prostate cancer metastasis}

The complex mechanism of metastasis and its regulation in human carcinomas is only beginning to be understood. For successful dissemination and metastatic growth a tumor cell needs to overcome several obstacles. The cell has to detach from the local primary tumor tissue architecture and, subsequently, invade the surrounding tissue, leading to invasive growth. Motile cells then have to enter the bloodstream, to intravasate, and travel in the vascular network to reach a distant location. Then the cell must be able to leave the bloodstream and invade into the target tissue and proliferate at the target site. Homing of the tumor cells at distant tissues is mainly regulated by the microenvironment of the target tissues. The production of growth factors and chemokines of distant tissues in combination with the expression of cell surface receptors on the tumor cell could result in the tumor cell's extravasation and ultimate settlement and proliferation in the target tissue. During the processes of 
migration, traveling and settling (Fig. 1), the cell must be able to survive independently outside of its normal environment.

The first step in metastasis is the detachment of the cells from the primary tumor and the gain of motility of those cells occurring between stage 1 and 2 (Fig. 1). As this is one of the first occurring differences between localized tumor cells and cells that gain metastatic potential, this process is a start to distinguish indolent tumors from their aggressive counterparts.

\section{Primary migration}

The epithelial cells adapt a migratory phenotype during the process of metastasis. The migration of epithelial cells is also involved in several organ genetic and morphogenetic processes during embryonic development. This migration, which is strictly regulated, shows parallels with the metastatic process of carcinomas (Grunert et al. 2003; Huber et al. 2005). One of the possible mechanisms of primary migration in embryonic development is proposed as the 'epithelial to mesenchymal transition' (EMT) of epithelial cells (Thiery 2002). In EMT epithelial cells shift from a rigid and steady phenotype to a highly motile phenotype, and as such, EMT merely is new nomenclature for one of the key steps in the invasive process. One of the characteristics of EMT in embryonic development is the loss of expression of cell adhesion molecules, filament assembly and the expression of extra cellular proteins like fibronectin and/or collagen (Boyer et al. 2000). However, the exact nature and regulation of a similar transition in carcinomas has not yet been fully elucidated.

Although a process like EMT may be involved during the first stages of carcinoma metastasis, this process is not the only possible mechanism of action. Another mechanism of carcinoma cell motility is a process called collective motility. In this process of invasion a collection of cells are moving from the primary tumor without individual de- attachment of the cells (Sahai 2005). The search for markers is not limited to one single process, as it is yet un-identified by which process prostate carcinoma cells are gaining invasive properties. Markers for EMT alone are not sufficient to detect all cells with invasive properties, as EMT may not be the only process involved. A combination of markers involved in distinctive processes seems to be a more promising approach.

In general, the primary migration of carcinoma cells is divided into three different events; loss of cell-cell adhesion, (partial) degradation of the extra cellular matrix (ECM) and actin/cytoskeletal changes within the cell. During an EMT-like mechanism all these processes occur while during collective motility the loss of cell-cell adhesion only takes place at specific sites. This occurs likely at the leading edge of the motile complex of cells, rather than on each individual cell. Detecting the loss of certain adhesion molecules or the gain of molecules corresponding to cell-motility is therefore not straightforward. A combination of markers involved in these processes may give insight into the complex mechanism of invasion. Furthermore, expression changes of these markers may reflect the tumors metastatic potential. An overview of those markers is given in Table 1 .

\section{Cell adhesion}

The epithelial cells of the prostate are categorized into three types; the basal cells, the luminal epithelial cells and the secretory cells. The basal epithelial cells are the first to appear during normal human prostate development. These cells subsequently undergo differentiation into intermediate and secretory cells. The epithelial cells however, become less differentiated during prostate carcinoma progression. Cells appear with features of both basal and luminal cells (Knudsen and Miranti 2006). The epithelial cells in the prostate gland are bound to one another as well as to the extracellular matrix (ECM). In the normal human prostate,

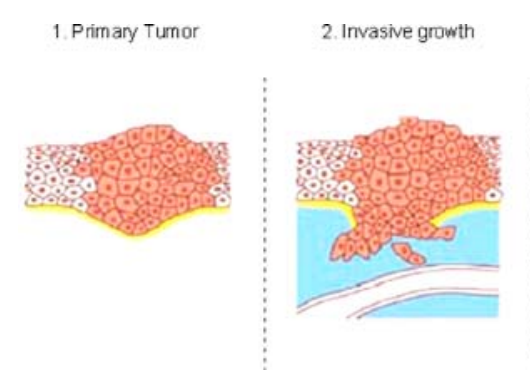

Fig. 1 Different stages of tumor metastasis presented in a schematical way. Stage 1 the primary tumor. There are no signs of migratory action. Stage 2 invasive growth. Cells de-attach from the primary tumor and start to invade the surrounding tissue. Stage 3 intravasation. Migratory cells reach the nearest blood vessel and intravasate into the blood

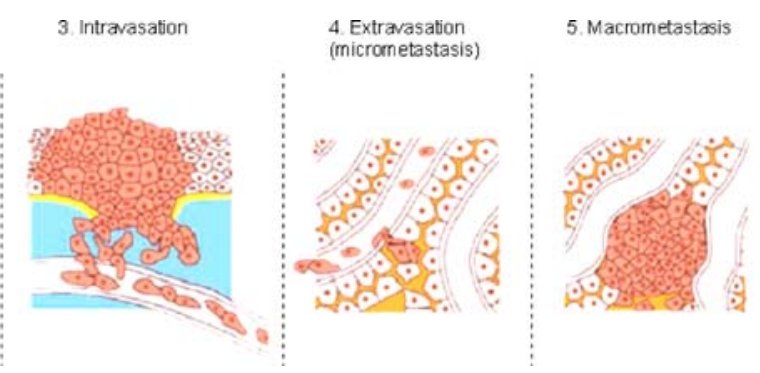

stream. Stage 4 extravasation. Traveling cells escape from the vessel and start to invade secondary tissues and micrometastasis of single surviving cells develops. Stage 5 macrometastasis. Micrometastasis progresses to macrometastasis, a secondary tumor has developed (adapted from Huber et al. 2005) 
Table 1 Potential markers involved in the early metastatic processes of prostate cancer

\begin{tabular}{|c|c|c|c|c|}
\hline Biological process & Marker & $\begin{array}{l}\text { Expression } \\
\text { Up } \uparrow / \text { down } \downarrow\end{array}$ & $\begin{array}{l}\text { First change } \\
\text { in expression }\end{array}$ & References \\
\hline \multirow[t]{3}{*}{ Cell-cell adhesion } & E-cadherin & $\downarrow$ & PC: Gleason Score 7-10 & $\begin{array}{l}\text { Rubin et al. (2001), } \\
\text { Jaggi et al. ( 2005), } \\
\text { Rhodes et al. ( 2003) }\end{array}$ \\
\hline & $\mathrm{N}$-cadherin & $\uparrow$ & PC: Gleason Score 7-10 & Jaggi et al. (2006) \\
\hline & Membranous $\beta$-catenin & $\downarrow$ & PIN & $\begin{array}{l}\text { Horvath et al. (2005) } \\
\text { Jaggi et al. (2005) }\end{array}$ \\
\hline Wnt Signaling & Nuclear $\beta$-catenin & $\uparrow$ & PC: Gleason Score 4-10 & Horvath et al. (2005) \\
\hline Cell-ECM Adhesion & $\beta 4$-integrin & $\downarrow$ & PIN & Davis et al. (2001) \\
\hline Integrin signaling/cell motility & FAK & $\uparrow$ & PIN & Rovin et al. (2002) \\
\hline \multirow{3}{*}{$\begin{array}{l}\text { Intercellular } \\
\quad \text { signaling/cell-cell adhesion }\end{array}$} & CX26 & $\uparrow$ & PC: Gleason Score $6(3+3)$ & Tate et al. (2006) \\
\hline & CX43 & $\downarrow$ & PC: Gleason Score 5-10 & Habermann et al.(2002) \\
\hline & CX32 & $\downarrow$ & PC: Gleason Score 5-10 & Habermann et al. (2002) \\
\hline \multirow[t]{2}{*}{ ECM Degradation } & MMP-2 & $\uparrow$ & PC: Gleason Score >5 & $\begin{array}{l}\text { Trudel et al. (2003); } \\
\text { Wood et al. ( 1997) }\end{array}$ \\
\hline & MMP-9 & $\uparrow$ & PC: Gleason Score >5 & $\begin{array}{l}\text { Trudel et al.(2003) } \\
\text { Wood et al.(1997) }\end{array}$ \\
\hline
\end{tabular}

Shown are the biological processes in which the markers are involved, the name of the markers, the change in expression, and the tissues in which the first significant change in expression of the marker was observed and references

ECM Extracellular matrix, PIN prostatic intraepithelial neoplasia, $P C$ prostate carcinoma, $F A K$ focal adhesion kinase, $C X$ connexin, $M M P$ Matrix metalloproteinase

the basal cells are the only cells of the prostate gland attaching to the substratum. However, less differentiated cells infiltrate into the basal cell layer in invasive carcinoma, indicating a change in cell adhesion.

Cell-cell adhesion is mediated by different junction protein complexes, while binding to the ECM is mainly regulated by integrins. The loss of cell-cell adhesion together with the gain of cell-ECM interactions, either transient or permanent, is an indispensable process when the carcinoma progresses to an invasive state (Stewart et al. 2004). Proteins involved in altered cellular adhesion properties may, therefore, function as biological markers.

\section{Cell-cell adhesion}

Cell-cell adhesion is mediated by adherens and tight junctions between epithelial cells. During the metastatic process of epithelial cells, both the composition of these cell junctions and the number of junctions change. Cell adhesion molecules (CAMs), like E-cadherin and $\mathrm{N}$-cadherin as well as $\beta$-catenin, are proteins, which are structurally involved in those junctions.

\section{E-cadherin}

E-cadherin, which is a member of the cadherin family of CAMs, mediates lateral cell-cell adhesion in secretory tissues, like the prostate. E-cadherin is a type- $1 \mathrm{Ca}^{2+}$-dependent cell adhesion molecule and is a major component of adherens junctions in epithelial cells. E-cadherin is located at the cell membrane. It facilitates the binding to different catenins $(\alpha-, \beta$ - and $\gamma$-catenin) that associate with actin filaments and the actin cytoskeleton within the cell.

The expression of E-cadherin has been extensively studied using tissue micro array (TMA) in prostate tumor, derived from radical prostatectomy (RP) (Rubin et al. 2001). In low-grade carcinomas with negative surgical margin, benign and normal tissue E-cadherin was normally expressed. The expression of E-cadherin was decreased in higher grade carcinomas and carcinomas with a positive surgical margin. Furthermore, metastatic tissues showed strong E-cadherin staining. This is rather controversial, as the related primary tumors showed loss of E-cadherin expression. The re-expression of E-cadherin in metastasis may imply that the loss of E-cadherin is a transient event occurring during invasion and diapedesis. The reexpression of E-cadherin in metastatic tissue may be regulated by paracrine signaling from cells of the metastatic environment.

The low expression of E-cadherin has been correlated with prostate specific antigen recurrence in serum after RP, suggesting that aberrant E-cadherin expression could be predictive of clinical outcome (Rhodes et al. 2003). The expression of E-cadherin is inversely correlated to tumor grade, even within one RP specimen containing both benign and malignant tissues (Jaggi et al. 2005). This also underlines the difficulty of E-cadherin expression analysis using prostate biopsies compared to PC specimens. Most 
prostate tumors are multi-focal and heterogeneous. During sampling of prostate biopsies, foci of malignant carcinoma within the prostate could be missed.

Most studies used a large number of samples (ranging from 16 to 259) to analyze the correlation of E-cadherin expression and tumor grade. Although a difference could be seen between the two groups of samples (high- vs. low Gleason score), not all individual cases showed a low E-cadherin expression in combination with a high Gleason score, indicating that E-cadherin expression may not be used as an individual marker. However, we may use E-cadherin expression data in combination with other markers of metastasis.

Besides decreased expression of E-cadherin, various studies were directed to the role of E-cadherin gene (CDH1) polymorphisms (Bonilla et al. 2006; Li et al. 2006; Verhage et al. 2002). A -160 C/A single nucleotide polymorphism was found in allele A. (Liet al. 2006). This polymorphism showed approximately $70 \%$ decreased transcription of allele A. This may be a cause of the decreased E-cadherin expression as observed in prostate carcinoma. The suitability of this polymorphism in predicting prostate carcinoma metastasis is, however, controversial. An elevated risk for prostate carcinoma was observed in allele A carriers in a Dutch population, with a higher risk for sporadic cancers (approximately fivefold) compared to hereditary cancer (approximately twofold) (Verhage et al. 2002). This is contradictory to Swedish results, which showed a higher risk for hereditary cancers (Jonsson et al. 2004). More $\mathrm{CDHl}$ polymorphisms were studied in AfricanAmericans, Jamaicans and European-American men (Bonilla et al. 2006). This study showed that a combination of polymorphisms $(160 \mathrm{~A}$ and $+54 \mathrm{~T}$ ) might present a susceptibility for prostate cancer in European populations.

The discrepancy between results of various studies reveals that more research must be done to link $\mathrm{CDH} 1$ polymorphisms to metastatic disease in prostate cancer. It is suggested that polymorphisms decrease E-cadherin transcription and thereby its expression. Therefore, E-cadherin protein expression itself, instead of gene polymorphisms, may be a valid marker. It could be even more promising to use E-cadherin protein expression data in combination with other markers.

\section{$N$-cadherin}

$\mathrm{N}$-cadherin is just like E-cadherin a type-I cadherin. While E-cadherin is mostly expressed in epithelial cells, N-cadherin is expressed in various cell types including nerve, myocardial and mesenchymal cells. Increase in N-cadherin expression is one of the features of epithelial to mesenchymal transition (EMT), together with loss of E-cadherin expression. The expression of E-cadherin declines, while
$\mathrm{N}$-cadherin expression increases during the process of EMT. N-cadherin facilitates a more dynamic cell-cell adhesion. $\mathrm{N}$-cadherin expression in prostate cancer is less well studied compared to E-cadherin expression. In one study, the switch of cadherin expression was associated with higher grade tumors (Jaggi et al. 2006). In a total of 44 PC specimens $45 \%$ showed $\mathrm{N}$-cadherin expression. Only $7 \%$ of the Gleason 5-6 tumors showed positive expression, compared to $65 \%$ of Gleason grade 7, and $57 \%$ of Gleason score 8-10. Although N-cadherin expression correlates with Gleason score, no data are available correlating Ncadherin expression to PSA recurrence, metastasis or tumor progression.

\section{$\beta$-catenin}

$\beta$-Catenin is a multifunctional protein; it is not only involved structurally in the adherens junction complex, but it also acts as signaling molecule. It connects the cytoplasmic part of E-cadherin to the cytoskeleton together with $\alpha$ catenin in the adherens junctions. When adherens junction complexes are lost, a soluble form of $\beta$-catenin becomes localized in the cytoplasm and some free $\beta$-catenin is transported to the nucleus. The cytoplasmic expression of $\beta$-catenin is rather low in normal cells as it is easily targeted for ubiquitination by GSK- $3 \beta$ phosphorylation and subsequently degraded by proteosomes. However, signaling by the Wnt pathway represses the degradation of $\beta$-catenin by degrading the GSK-3 $\beta / \beta$-catenin complex. This leads to suppressed phosphorylation of $\beta$-catenin and, consequently, accumulation of the protein. The Wnt signaling might be involved in prostate cancer development (Yardy and Brewster 2005). Besides the Wnt signaling pathway the Pi-3K/Akt pathway also seems to be involved in regulating the soluble pool of free $\beta$-catenin. The Pi-3K/Akt pathway signaling is also involved in the development of prostate carcinoma, mainly by loss of the PTEN Akt suppressor (Downward 2004).

The higher levels of soluble $\beta$-catenin may lead to higher amounts of this protein in the nucleus were it exerts its transcriptional function. It interacts with different DNA-binding transcription factors and especially the TCF/lymphoid enhancer factor (LEF) family, which includes TCF1, TCF3-4 and LEF1. Those DNA-binding proteins repress the transcription of target genes by binding transcriptional repressors. $\beta$-catenin competes for binding with those repressors, leading to transcriptional activation of the target genes. The transcriptional activation of $\beta$-catenin (CRT) is involved in numerous processes in normal cells, from embryonic anterior-posterior axis specification to tissue development. Hyper-activation of CRT signaling may elicit pronounced morphology and trans-differentiation in prostatic neoplasia (Chesire et al. 2002). Although the loss of adherens junctions leads to accumulation of free soluble 
$\beta$-catenin it does not necessarily result in higher activation of $\beta$-catenin signaling as free $\beta$-catenin is rapidly degraded. However, in combination with ubiquitination repression, $\beta$-catenin accumulation could be, partially, responsible for prostate carcinoma progression due to elevated CRT signaling.

Normal prostate tissue shows high membranous $\beta$-catenin expression and low nuclear $\beta$-catenin staining. Prostatic intraepithelial neoplasia (PIN) lesions show less expression of membranous $\beta$-catenin. This indicates that the loss of adherens junctions is an event occurring early in tumor development, assuming PIN lesions as precursors of prostate carcinoma.(Jaggi et al. 2005) However, the loss of membranous $\beta$-catenin occurs at a very low rate and does not correlate with tumor stage or grade. The loss of membranous $\beta$-catenin could be a direct consequence of loss of adherens junctions early in tumor development.

Membranous $\beta$-catenin was lowered in both benign prostate hyperplasia (BPH), a non-malignant enlargement of the prostate, and localized prostate cancer (Horvath et al. 2005). Nuclear $\beta$-catenin staining is stronger in BPH compared to normal prostate tissue. This indicates that $\beta$-catenin accumulation in the cytoplasm, due to loss of membranous $\beta$-catenin, leads to higher nuclear levels. However, when compared with localized prostate cancer, BPH had higher levels of nuclear staining. Advanced prostate cancer showed even less nuclear $\beta$-catenin staining compared with localized disease. Furthermore, lower levels of nuclear $\beta$-catenin correlate with poorer prognosis (Horvath et al. 2005).

$\beta$-catenin signaling may both, repress and promote tumor growth. Higher nuclear $\beta$-catenin levels indicate tumor repression while lower nuclear $\beta$-catenin levels indicate tumor growth. The stabilization of nuclear $\beta$-catenin levels is regulated by various cofactors and the balance between those factors and $\beta$-catenin results in tumor promotion, or repression. The fact that relatively low nuclear $\beta$-catenin levels do correlate with disease outcome suggests nuclear $\beta$-catenin as a possible marker. More studies of low-risk prostate cancer may clarify the role of nuclear $\beta$-catenin levels during prostate cancer progression.

\section{Cell-ECM adhesion/focal adhesions}

Besides cell-cell adhesion, epithelial cells are also connected to the ECM. The interaction with the ECM is indispensable for the traveling of a metastatic cell. The interaction with the ECM changes when the cell has metastatic potential. Integrins may be involved in such changes.

Integrins are important mediators in the attachment of epithelial cells to the ECM. These appear in complexes at the cell surface known as focal adhesions. Integrins play an important role in tumor-associated signaling events besides anchoring to the ECM. Focal adhesion kinase (FAK) is an important mediator of integrin signaling. Integrin-dependent signaling is supposed to affect cell growth, anchoragedependent differentiation, adhesion, motility and apoptosis.

\section{Integrins}

Integrins appear in heterodimeric structures containing an $\alpha$ and $\beta$ chain. The predominant structures found on epithelial cells are the $\alpha 5 \beta 1, \alpha 6 \beta 1$ and the $\alpha 6 \beta 4$ integrins. The suprabasal and secretory cells are distinct from the basal cells in the expression of integrins and adhesion molecules that are able to connect to the substratum. In normal prostate glands, only the basal cells express integrins connecting them to the substratum. The $\alpha 6 \beta 4$ integrin, for example, is an important component of the hemidesmosome expressed at the basal surface in most stratified epithelial cells. The hemidesmosome links the cytoskeleton intermediate filaments to laminin-5 in the ECM.

Basal epithelial cells are lost during PC progression. Partial loss of the basal lamina is a hallmark of high-grade PIN. The basal cell lining almost completely disappears in PC. The expression of integrins changes together with the loss of the basal lining. The $\beta 4$ integrin, for example, was lost in PIN lesions together with basal cell-lining and in prostate carcinoma the expression of $\beta 4$ integrins was totally lost (Davis et al. 2001). Although the $\beta 4$ integrin was lost, the $\alpha 6 \beta 1$ integrin showed to be continuously expressed through all cancer stages, which may indicate that the composition of integrins changes during tumor progression.

Expression of laminin-5, the major ligand of $\alpha 6 \beta 4$ integrin, is also declining through PC progression (Davis et al. 2001). This may indicate that the composition of the local ECM is rather important for physiology. The continuous expression of the $\alpha 6 \beta 1$ integrin together with the loss of $\alpha 6 \beta 4$ integrin may reflect the motility of the cancer cells. Loss of the $\alpha 6 \beta 4$ integrin may give a less-stable attachment to the ECM, while the continued expression of the $\alpha 6 \beta 1$ integrin may provide enough attachment for the cells to become mobile. This may also explain why prostate cancer cells favor to move along laminin-5 coated nerves (Cress et al. 1995).

The composition of integrins in prostate cancer tissue, like the combination of loss of $\alpha 6 \beta 4$ and continuous expression of $\alpha 6 \beta 1$, may reflect the capability of the cells to metastasize. Such a combination may, therefore, be a valid biological marker.

\section{Focal adhesion kinase}

Signaling of integrins is mediated by the connection of integrins to focal adhesion kinase (FAK). FAK, a protein 
tyrosine kinase, is localized at focal adhesions at the cell membrane and signals through the phosphoidyllinositol 3 kinase (PI 3) pathway. This integrin-dependent signaling is, in general, known to regulate several tumor-progressing processes as cell growth, adhesion-based differentiation, adhesion, motility and apoptosis (Downward 2004). Increasing levels of FAK in human prostate cancer cell lines correlate with greater metastatic potential (Tremblay et al. 1996). FAK was predominantly expressed in the basal epithelial cell layer as shown in an immunohistochemical analysis of various prostate specimens (Rovin et al. 2002). FAK overexpression appeared in PIN lesions, with a clear distribution of high FAK staining in neoplastic cells while normal cells in the surrounding normal tissues did not show elevated expression. Furthermore, benign prostate hyperplasia (BPH) did not show a change in FAK expression compared to normal tissue. Beside the FAK overexpression in PIN lesions, higher grade and metastatic carcinomas retained the elevated FAK expression, suggesting an important role for FAK in tumor progression (Rovinet al. 2002). The elevated expression of FAK in early stage carcinoma may, therefore, be used as a biological marker.

\section{Gap junctions and gap junctional intercellular communication (GJIC)}

Comparing tight and adherens junctions with gap junctions, the latter is used for intercellular communication rather than anchoring to neighboring cells. Gap junctions are intra-cellular membrane channels composed of a pair of hemi-channels (connexons), which are formed by oligomerization of six protein sub-units, which are called connexins. The connexons align to the connexons of an adjacent cell creating an intracellular channel. These channels are able to transport ions and small molecules $(<1.5 \mathrm{kDa})$ between cells. This intercellular communication is termed gap junctional intercellular communication (GJIC), and is involved in tissue homeostasis, growth control, differentiation and apoptotic events. Gap junction formation has been reported to decrease as cancer progresses. As connexins are the major components of the gap junctions, a change in connexin expression may serve as indicator for cancer progression.

\section{Connexins}

Of the existing connexins only CX43 and CX32 and CX26 are known to be expressed in endocrine and exocrine glands. In normal prostate tissues CX32 is punctually expressed in the luminal cells on cell borders but also diffuse in the cytoplasm. CX43 is punctually expressed mainly in the basal cell compartment on cell borders. As most of the epithelial cells in the prostate glands are luminal cells, detecting CX43 is difficult.

CX43 and CX32 have been studied in prostate tissues. In normal tissue and BPH both connexins were expressed. However, in some of the samples only one of the connexins was expressed. Not even one tissue specimen was negative for both connexins. BPH tissue showed stronger connexinstaining compared with normal tissue. In prostate carcinoma tissue the expression of connexins was less compared with BPH and normal tissue. In addition, some samples showed loss of both connexins (Habermann et al. 2002).

In a study of prostate cancer cell line (LNCaP and PC-3) progression models it was found that CX26 and CX43 were overexpressed (Tate et al. 2006). This finding conflicts with previous clinical findings (Mesnil 2002). However, the overexpression of CX26 was confirmed by immunohistochemical analysis of clinical specimens. In samples with a high Gleason score $(3+3) 66 \%$ stained moderately to intensively for CX26. In benign tissues, only $29 \%$ had a similar expression (Tate et al. 2006). This overexpression seems to be unrelated to the gap junction formation, as CX26 was not localized to gap junction structures in investigated cell-line models. Furthermore, immunoprecipitation showed that CX26 complexes with focal adhesion kinase (FAK). FAK is a kinase, which interacts with activated integrins and affects the cytoskeleton to improve motility, discussed earlier in this review.

The role of connexins in tumorigenesis is still largely unknown; however, the aberrant expression of connexins may indicate that the loss of GJIC is involved in the pathogenesis of prostate cancer. Furthermore, the overexpression of CX26 and its interaction with focal adhesion kinase suggests that connexins could be involved in tumor progression, independent of gap junction formation. So the individual gain of connexin expression may be a valid marker for tumor progression.

\section{Extracellular matrix (ECM) degradation}

A cell has to travel through the extracellular matrix (ECM) as soon as it becomes invasive. The ECM must be partially degraded to allow the cells' passing. Matrix metalloproteinases (MMPs) are the only known enzymes, which are able to degrade the ECM as well as the basement membrane and so they are thought to be important mediators during tumor metastasis.

\section{Matrix metalloproteinase (MMPs)}

The matrix metalloproteinases are endopeptidases, which include approximately 20 zinc-dependent proteinases that degrade several components of the ECM like collagen, 
proteoglycans, and glycoproteins. Of the different types of MMPs the type IV collagenases are thought to be involved in prostate-cancer invasion. Type IV collagenases are able to degrade collagen, thereby facilitating the penetration of the basement membrane. MMP2 and MMP9 are both extensively studied. Overexpression of both MMP2 and MMP9 is correlated with a higher Gleason score and invasive phenotypes (Wood et al. 1997). Furthermore, overexpression of MMP2 is negatively correlated with disease-free survival(Trudel et al. 2003).

MMPs are mainly produced by stromal cells. The produced MMPs occur in their inactive form and become activated by other MMPs. For example, MMP2 is produced as the inactive pro-MMP-2. This soluble form is cleaved by MMP14 to produce the active MMP-2. The active forms of MMP-2 and MMP-13 in their turn are able to activate MMP-9. This active form is able to bind to the integrins outside of the cancer epithelial cells to perform its function. This also implicates difficulties in measuring expression of the active MMPs. Only the bound MMPs form must be taken in account, which means that a good distinguishing must be made between normal versus malignant cells within the tissue sample.

Both MMP-2 and MMP-9 overexpression is implicated as prognostic marker in prostate cancer, together with downregulation of their inhibitors; the tissue inhibitors of metalloproteinases (TIMPs) (Morgia et al. 2005). Furthermore, the mRNA ratio between MMP9 and E-cadherin showed to be an important indicator for clinical outcome after radical prostatectomy(Kuniyasu et al. 2003). This implies that the expression of metalloproteinases could be used as biological marker in combination with other markers involved in the metastatic process.

\section{Multiple marker approach}

The search for new prognostic markers in prostate cancer is increasingly becoming a field of interest since the last decade. Most researchers aimed at finding one single marker. They focused on the difference in expression between patient groups with low-aggressive carcinomas versus groups with high-aggressive carcinomas. Such a strategy revealed a significant correlation between a change in expression of the single marker and disease development in a number of studies. However, this correlation merely indicates a role of the discussed marker in tumor development; it does not indicate the actual disease process in an individual patient.

In view of the complexity of the prostate tumor pathology the success of searching for one single marker seems very small. Tumors of the prostate are heterogeneous, not only presenting multiple tumor foci but also a heterogeneous appearance within one single tumor. For a prostate tumor to become metastatic and dangerous, multiple mechanisms must be activated: from loss of cell adhesion to increasing cell motility as well as a change in the direct tumor environment. Not all of these mechanisms appear at the same time and some processes are even transient. Focusing on one single marker may therefore not be sufficient. To study a cohort of markers representing multiple biological processes may give a better indication for tumor progression than one single marker could.

\section{Concluding remarks}

In this review potential markers, which represent essential biological functions within the first step of tumor progression were discussed: gaining metastatic potential. E-cadherin, $\mathrm{N}$-cadherin, $\beta$-catenin, integrins, focal adhesion kinase (FAK), connexins and metalloproteinases (MMPs) are markers that represent cell-cell adhesion, cell-ECM interaction and ECM degradation.

Today, there is still little knowledge of the clinical value of the discussed mechanisms. Creating a model of concerted expression of markers involved in those mechanisms and analyzing their correlation with disease progression, may provide more insight into the early biological processes of metastasis and their clinical values. When expression analysis of markers in a cohort of tumor samples is performed, a possible prediction scheme could be generated for individual cases. In such an analysis not only the expression of one single marker but rather the expression ratio between multiple markers is expected to be of more prognostic value.

A concerted expression model of a combination of markers, representing aggressiveness in early stage prostate cancer could have important clinical impact by providing a more efficient approach towards diagnosis and treatment of early-stage prostate cancer.

\section{References}

Bonilla C, Mason T, Long L et al (2006) E-cadherin polymorphisms and haplotypes influence risk for prostate cancer. Prostate 66:546-556

Boyer B, Valles AM, Edme N (2000) Induction and regulation of epithelial-mesenchymal transitions. Biochem Pharmacol 60:1091-1099

Chesire DR, Ewing CM, Gage WR, Isaacs WB (2002) In vitro evidence for complex modes of nuclear beta-catenin signaling during prostate growth and tumorigenesis. Oncogene 21:2679-2694

Crawford ED (2003) Epidemiology of prostate cancer. Urology 62:3-12

Cress AE, Rabinovitz I, Zhu W, Nagle RB (1995) The alpha 6 beta 1 and alpha 6 beta 4 integrins in human prostate cancer progression. Cancer Metastasis Rev 14:219-228

Davis TL, Cress AE, Dalkin BL, Nagle RB (2001) Unique expression pattern of the alpha6beta4 integrin and laminin-5 in human prostate carcinoma. Prostate 46:240-248 
Downward J (2004) PI 3-kinase, Akt and cell survival. Semin Cell Dev Biol 15:177-182

Grunert S, Jechlinger M, Beug H (2003) Diverse cellular and molecular mechanisms contribute to epithelial plasticity and metastasis. Nat Rev Mol Cell Biol 4:657-665

Habermann H, Ray V, Habermann W, Prins GS (2002) Alterations in gap junction protein expression in human benign prostatic hyperplasia and prostate cancer. J Urol 167:655-660

Horvath LG, Henshall SM, Lee CS et al (2005) Lower levels of nuclear beta-catenin predict for a poorer prognosis in localized prostate cancer. Int J Cancer 113:415-422

Huber MA, Kraut N, Beug H (2005) Molecular requirements for epithelial-mesenchymal transition during tumor progression. Curr Opin Cell Biol 17:548-558

Jaggi M, Johansson SL, Baker JJ, Smith LM, Galich A, Balaji KC (2005) Aberrant expression of E-cadherin and beta-catenin in human prostate cancer. Urol Oncol Semin Original Invest 23:402-406

Jaggi M, Nazemi T, Abrahams NA, Baker JJ, Galich A, Smith LM, Balaji KC (2006) N-cadherin switching occurs in high Gleason grade prostate cancer. Prostate 66:193-199

Jonsson BA, Adami HO, Hagglund M, Bergh A, Goransson I, Stattin P, Wiklund F, Gronberg H (2004) -160C/A polymorphism in the E-cadherin gene promoter and risk of hereditary, familial and sporadic prostate cancer. Int J Cancer 109:348-352

Klotz L (2006) Active surveillance with selective delayed intervention for favorable risk prostate cancer. Urol Oncol Semin Original Invest $24: 46-50$

Knudsen BS, Miranti CK (2006) The impact of cell adhesion changes on proliferation and survival during prostate cancer development and progression. J Cell Biochem 99:345-361

Kuniyasu H, Ukai R, Johnston D, Troncoso P, Fidler IJ, Pettaway CA (2003) The relative mRNA expression levels of matrix metalloproteinase to E-cadherin in prostate biopsy specimens distinguishes organ-confined from advanced prostate cancer at radical prostatectomy. Clin Cancer Res 9:2185-2194

Li HC, Albert JM, Shinohara ET et al (2006) E-cadherin promoter polymorphisms are not associated with the aggressiveness of prostate cancer in Caucasian patients. Urol Oncol Semin Original Invest 24:496-502

Mesnil M (2002) Connexins and cancer. Biol Cell 94:493-500

Morgia G, Falsaperla M, Malaponte G, Madonia M, Indelicato M, Travali S, Mazzarino MC (2005) Matrix metalloproteinases as diagnostic (MMP-13) and prognostic (MMP-2, MMP-9) markers of prostate cancer. Urol Res 33:44-50
Rhodes DR, Sanda MG, Otte AP, Chinnaiyan AM, Rubin MA (2003). Multiplex biomarker approach for determining risk of prostatespecific antigen-defined recurrence of prostate cancer. J Natl Cancer Inst 95:661-668

Rovin JD, Frierson HF Jr, Ledinh W, Parsons JT, Adams RB (2002) Expression of focal adhesion kinase in normal and pathologic human prostate tissues. Prostate 53:124-132

Rubin MA, Mucci NR, Figurski J, Fecko A, Pienta KJ, Day ML (2001) E-cadherin expression in prostate cancer: a broad survey using high-density tissue microarray technology. Human Pathol 32:690-697

Sahai E (2005) Mechanisms of cancer cell invasion. Curr Opin Genet Dev 15:87-96

Stamey TA, Johnstone IM, McNeal JE, Lu AY, Yemoto CM (2002) Preoperative serum prostate specific antigen levels between 2 and $22 \mathrm{ng} / \mathrm{ml}$ correlate poorly with post-radical prostatectomy cancer morphology: prostate specific antigen cure rates appear constant between 2 and $9 \mathrm{ng} / \mathrm{ml}$. J Urol 167:103-111

Stewart DA, Cooper CR, Sikes RA (2004) Changes in extracellular matrix (ECM) and ECM-associated proteins in the metastatic progression of prostate cancer. Reprod Biol Endocrinol 2:2

Tate AW, Lung T, Radhakrishnan A, Lim SD, Lin X, Edlund M (2006) Changes in gap junctional connexin isoforms during prostate cancer progression. Prostate 66:19-31

Thiery JP (2002) Epithelial-mesenchymal transitions in tumour progression. Nat Rev Cancer 2:442-454

Tremblay L, Hauck W, Aprikian AG, Begin LR, Chapdelaine A, Chevalier S (1996) Focal adhesion kinase (pp125FAK) expression, activation and association with paxillin and p50CSK in human metastatic prostate carcinoma. Int J Cancer 68:164-171

Trudel D, Fradet Y, Meyer F, Harel F, Tetu B (2003) Significance of MMP-2 expression in prostate cancer: an immunohistochemical study. Cancer Res 63:8511-8515

Verhage BA, van HK, Ruijter TE, Kiemeney LA, Schalken JA (2002) Single-nucleotide polymorphism in the E-cadherin gene promoter modifies the risk of prostate cancer. Int J Cancer 100:683-685

Wood M, Fudge K, Mohler JL, Frost AR, Garcia F, Wang M, Stearns ME (1997) In situ hybridization studies of metalloproteinases 2 and 9 and TIMP- 1 and TIMP-2 expression in human prostate cancer. Clin Exp Metastasis 15:246-258

Yardy GW, Brewster SF (2005) Wnt signaling and prostate cancer. Prostate Cancer Prostatic Dis 8:119-126 